\title{
Design and synthesis of novel silicon-containing small molecule peptidomimetics with nanomolar anticancer activities
}

\author{
Lajos Gera ${ }^{1}$, Peter Hegyes ${ }^{2}$, Daqing $\mathrm{Wu}^{3}$, Robert Hodges ${ }^{1}$ \\ ${ }^{1}$ University of Colorado Denver, Anschutz Medical Campus, Aurora, CO, 80045, United States \\ ${ }^{2}$ Avidin Kft, Szeged, H-6726, Hungary \\ ${ }^{3}$ Augusta University, Augusta, GA, 30912, United States
}

https://doi.org/10.17952/35EPS.2018.160

\section{Introduction}

Cancer is a major health problem around the globe. Each year, tens of millions of people are diagnosed with cancer and more than half of the patients eventually die from it. In 2018, 1,735,350 new cancer cases and 609,640 cancer deaths are projected to occur in the United States. Though there has been a steady increase in survival for most cancers, the death rate remains unacceptable for certain cancers, e.g. lung (26\%), prostate (9\%), colon (8\%), pancreas (7\%) and breast (14\%). In this study, we support the concept that the ideal drug should have a multi-targeted mechanism that affects several proteins or events that contribute to the etiology, pathogenesis and progression of disease. In addition, multi-pathway targeting is one of the strategies to overcome chemo-resistance.

\section{Results and Discussion}

To design our novel anticancer drugs with unique structural properties, we have taken an innovative and nontraditional approach where we combine pharmacophoric components to create new and highly potent small peptidomimetic molecules with a simple three component A-B-C structure where each pharmacophore is known to have anticancer properties on its own or when incorporated into a multicomponent small molecule drug. Recently, we developed a new generation of this simple 3-componentA-B-C structure, a highly potent anticancer compoundGH501 [1] (Figure 1). In our new compounds,GH1501 - GH1504, the A-component was further modified to contain a silicon atom [2] (Figure 1). Using silicon over carbon has many advantages: a) compounds are more lipophilic than their carbon equivalent b) silicon can improve compound permeability c) silicon can lower compound toxicity d) silicon can change receptor selectivity e) silicon can enhance anticancer activity of compounds f) silicon compounds can treat drug-resistant tumors. The "A"-component 4-[3(trimethylsilyl)propoxy]benzoic acid (m.p.: 172-173 ${ }^{\circ} \mathrm{C}$, crystallized from $\mathrm{ACN}$ ) and 4-[butyldimethylsilyl)methoxy]benzoic acid (m.p.: $57-59{ }^{\circ} \mathrm{C}$ ) were synthesized via a modified Hegyes method using 4hydroxybenzoic acid methylester sodium salt. [3]. Interestingly, Zaltariov et al. [4] claimed that they synthesized 4[3-(trimethylsilyl)propoxy]benzoic acid for the first time. Also, their synthetic method provided only the 3(trimethylsilyl)propyl 4-hydroxybenzoate (m.p.: $114-117^{\circ} \mathrm{C}$ ) in our hands. The constituent at position A of our A-B-C structures is dependent on the composition of the B-component. When the B-component is 4-(2,6-dichloro-benzyloxy)phenyl the best silyl-alk oxy-benzoyl derivative is GH1501 (compare GH1501 and GH1503, Table 1). When the Bcomponent is biphenyl the best silyl-alkoxy-benzoyl derivative is GH1504 (compare GH1504 and GH1502, Table 1).

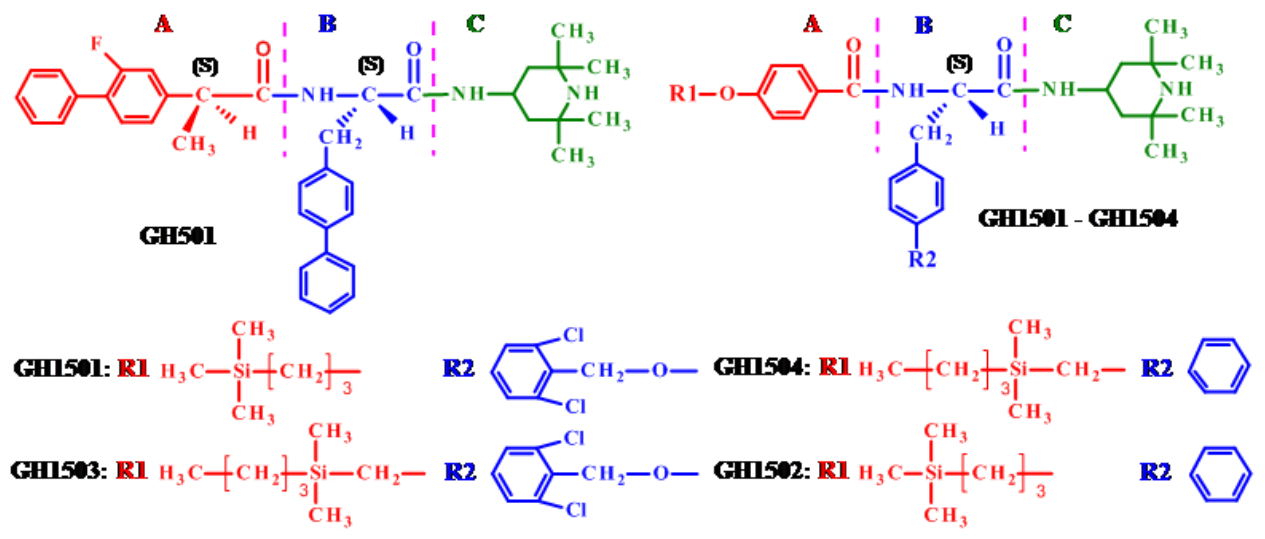

Figure 1: Structures of GH501 and GH1501 - GH1504 
Table 1: Results of the NCI human colon cancer cell line. GI50: concentration in nanomolar that inhibits cancer growth by 50\% Average: average of GI50 value for each compound Pink highlight is a GI50 value less than 500 $n M$ Yellow highlight is the average GI50 value less than $500 \mathrm{nM}$

\begin{tabular}{|c|c|c|c|c|c|}
\hline Cell Line & \multicolumn{5}{|c|}{ GIso (nanomolar) of selected anticancer compounds } \\
\hline Colon Cancer & GH501 & GH1501 & GH1503 & GH1504 & GH1502 \\
\hline COLO 205 & 184 & 216 & 342 & 215 & 392 \\
\hline HCC-2998 & 418 & 1050 & 1070 & 1110 & 1560 \\
\hline HCT-15 & 1440 & 357 & 323 & 309 & 437 \\
\hline HCT-116 & 145 & 206 & 319 & 218 & 297 \\
\hline HT29 & 178 & 263 & 342 & 315 & 430 \\
\hline KM12 & 461 & 363 & 382 & 376 & 413 \\
\hline SW-620 & 294 & 313 & 318 & 316 & 392 \\
\hline Average & 446 & 395 & 442 & 408 & 560 \\
\hline
\end{tabular}

GH501, GH1501GH1504

were

made

using

Bec-

chemistry

GI50: concentration in nanomolar that inhibits cancer growth by $50 \%$ Average: average of GI50 value for each compound Pink highlight is a GI50 value less than $500 \mathrm{nM}$ Yellow highlight is the average GI50 value less than $500 \mathrm{nM}$.

The diversity of the A-componentcan be Fluoro-biphenyl (GH501), silyl-alkoxy-benzoyl derivative (GH1501 or GH1504) depending on the "B"-component. The organo-silicon compounds showed cancer-type specific anticancer activity for colon cancer.

\section{Acknowledgements}

The authors are grateful to NCI for the NCI-60 human tumor cell line screening and to Eli Lilly for preliminary studies with our compounds on mechanism of action. We thank the NIH/NCI (Grant No. 1R41CA206725-01A1) for financial support.

\section{References}

1. Gera, L., at al. Proceedings 24th APS, p. 241-244 (2015).

2. Gera, L., Hodges, R.S., Hegyes, P. US Patent, US 9828393 B2, Nov. 28, 2017.

3. Hegyes, P., Toeroecsik, M. Hun. Pat. Appl. (2000), HU 9801407 A, March 28, 2000.

4. Zaltariov. M-F., et al. J. Mol. Struct. 1120, 302-316 (2016). 